\title{
Characterization of the collagen microstructural organization of human cervical tissue
}

\author{
Jia Hao ${ }^{1}$, Wang $\mathrm{Yao}^{2}$, W B Ryan Harris ${ }^{1}$, Joy Y Vink ${ }^{3}$, Kristin M Myers² and Eve Donnelly ${ }^{1,4}$ \\ ${ }^{1}$ Department of Materials Science and Engineering, Cornell University, Ithaca, New York, USA, ${ }^{2}$ Department of \\ Mechanical Engineering, Columbia University, New York, New York, USA, ${ }^{3}$ Department of Obstetrics and \\ Gynecology, Columbia University Medical Center, New York, New York, USA and ${ }^{4}$ Research Division, Hospital for \\ Special Surgery, New York, New York, USA
}

Correspondence should be addressed to E Donnelly; Email: eve.donnelly@cornell.edu

\begin{abstract}
The cervix shortens and softens as its collagen microstructure remodels in preparation for birth. Altered cervical tissue collagen microstructure can contribute to a mechanically weak cervix and premature cervical dilation and delivery. To investigate the local microstructural changes associated with anatomic location and pregnancy, we used second-harmonic generation microscopy to quantify the orientation and spatial distribution of collagen throughout cervical tissue from 4 pregnant and 14 non-pregnant women. Across patients, the alignment and concentration of collagen within the cervix was more variable near the internal os and less variable near the external os. Across anatomic locations, the spatial distribution of collagen within a radial zone adjacent to the inner canal of the cervix was more homogeneous than that of a region comprising the middle and outer radial zones. Two regions with different collagen distribution characteristics were found. The anterior and posterior sections in the outer radial zone were characterized by greater spatial heterogeneity of collagen than that of the rest of the sections. Our findings suggest that the microstructural alignment and distribution of collagen varies with anatomic location within the human cervix. These observed differences in collagen microstructural alignment may reflect local anatomic differences in cervical mechanical loading and function. Our study deepens the understanding of specific microstructural cervical changes in pregnancy and informs investigations of potential mechanisms for normal and premature cervical remodeling.

Reproduction (2018) 156 71-79
\end{abstract}

\section{Introduction}

Preterm birth (PTB) poses a worldwide, significant problem to both patients and physicians with an increased risk of neonatal death and a litany of associated longterm morbidities. Depending on the country of origin, the frequency of babies born before 37-week gestation can range from 7 to $15 \%$ of total births (Robert et al. 2008). Although there are numerous and complex etiologies that trigger spontaneous PTB (sPTB), the final common pathway must involve premature cervical remodeling, shortening and dilation for a fetus to be born premature. Because cervical tissue remodeling and dilation are required for vaginal delivery, a better understanding of normal cervical tissue structure and function is required before the pathophysiology that occurs in premature cervical remodeling can be discerned and diagnostic and preventative measures for SPTB can be identified.

Cervical tissue comprises (34-77\% dry weight) type I and type III collagen fibers, elastic fibers, various proteoglycans, hyaluronic acid, matricellular proteins and a population of cervical fibroblasts and smooth muscle cells (Myers et al. 2015a, Vink et al. 2016).
Cervical remodeling during pregnancy, in part, involves changes in collagen alignment and organization at multiple length scales (Fig. 1). At the molecular level, term pregnant (PG) tissue is associated with weaker collagen crosslinking compared to non-pregnant (NP) tissue, demonstrated by collagen solubility assays (Myers et al. 2009). At the millimeter-scale (mm-scale), an early x-ray diffraction study revealed that NP cervical tissue contains multiple radial zones of preferentially aligned collagen: (1) a middle zone of collagen fibers preferentially aligned around the endocervical canal and (2) an inner and outer zone of collagen fibers parallel to the canal (Weiss et al. 2006). Recently, a fiber tracking analysis at the mm-scale using optical coherence tomography (OCT) confirmed the existence of a middle zone of fibers circumferentially oriented around the endocervical canal, with the zone extending to the outer edge of the cervix (Yao et al. 2016). A distinct inner zone with collagen fibers preferentially aligned perpendicular to the endocervical canal was also identified. Overall, compared to PG cervical samples, NP samples have collagen fibers with greater alignment and lower dispersion, a measure of the 


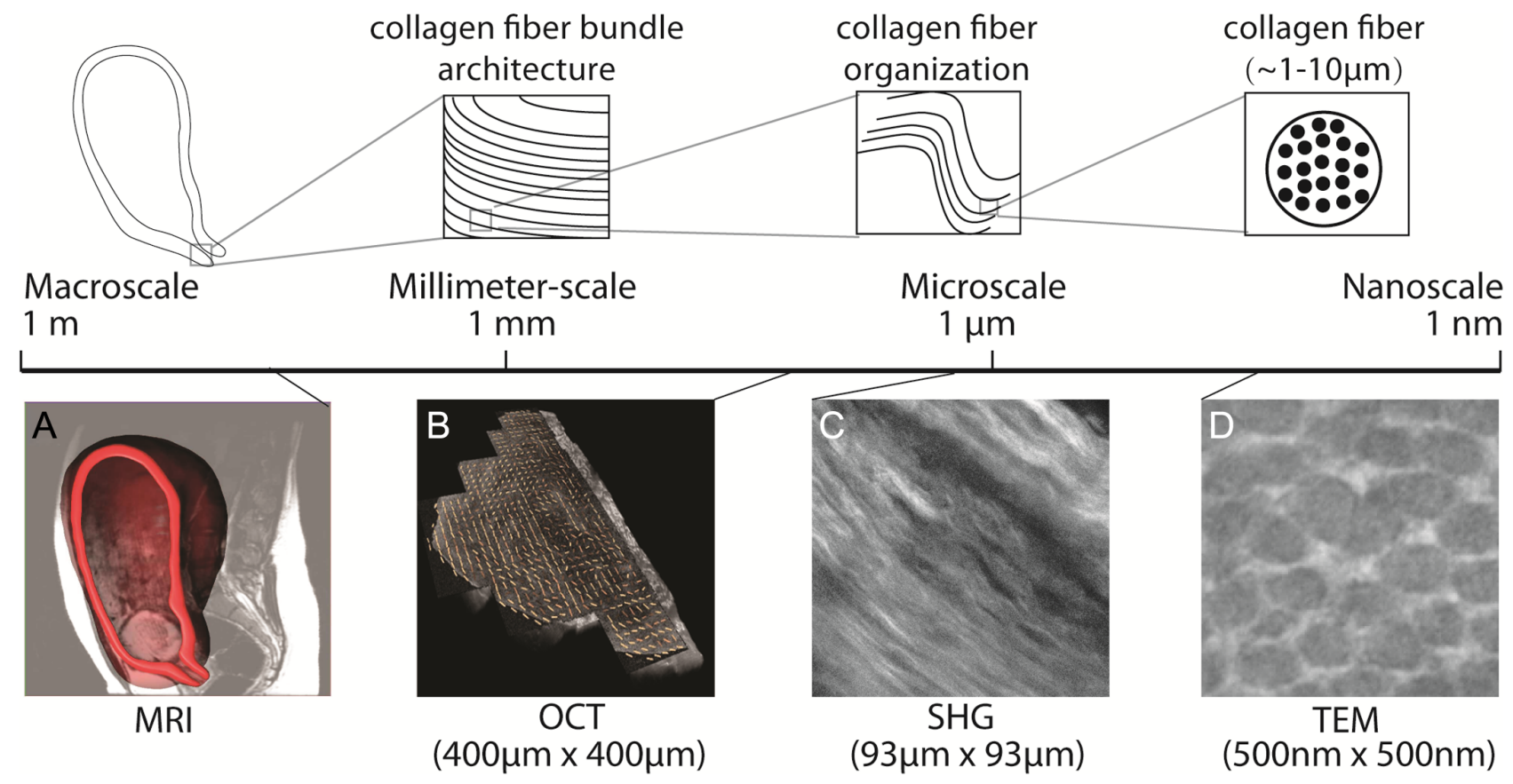

Figure 1 Length scales of cervical structure. (A) Macroscale structure $(\sim \mathrm{cm}-\mathrm{m})$ is shown in magnetic resonance images. (B) Millimeter-scale structure is shown in optical coherence tomography images of collagen fiber bundle architecture. (C) Microscale structure is shown in SHG microscopy images of collagen fiber organization. (D) Nanoscale collagen fiber structure is shown in TEM images. OCT, optical coherence tomography; SHG, second-harmonic generation; TEM, transmission electron microscopy. Adapted from Myers et al. (2015a); license 4146861173273 .

off-axis spread of fibers about the main direction. For NP samples, the posterior and anterior quadrants of the outer zone had more-aligned fibers compared to the other anatomic zones. Together, these data establish the existence of regional differences in collagen structure at the $\mathrm{mm}$-scale in cervical tissue.

The mechanical function of the cervix is thought to rely on the organization and alignment of its hierarchal collagen network (Fernandez et al. 2016). The mechanical properties of cervical tissue depend on the direction of loading, which is postulated to be attributed to the alignment of the collagen fiber network on the mm-scale (Myers et al. 2010, 2015b). Mechanical tests have demonstrated that term PG tissue is drastically softer and more compliant than NP tissue, where overall stiffness declines by two to three orders of magnitude (Myers et al. 2010, 2015b). Preliminary material modeling suggests the level of mechanical softening required for parturition cannot be achieved by the disorganization of the overall mm-level alignment of the collagen network alone, but instead by the remodeling of the collagen fiber itself (Yoshida et al. 2016). These structure-function relationships at the various length scales offer a potential biomarker for women at risk for premature cervical remodeling, where changes in the collagen structure may potentially serve as a diagnostic indicator of a mechanically soft cervix (Zhang et al. 2012).
Despite considerable evidence that the collagen network dramatically changes in pregnancy, the 'microstructure' of the collagen network (defined as the organization of the collagen network on a micron scale) in NP and PG human cervices is yet to be quantitatively characterized. In addition, with the introduction of cervical length screening, clinically, we see regional differences in cervical tissue function. For instance, in women who have premature cervical shortening, we commonly see that the internal os (the upper part of the cervix that meets the uterus) starts to dilate or funnel first while the external os (the lower part of the cervix that opens into the vagina) remains closed (Mancuso et al. 2010). Biomechanical computational models of pregnancy also confirm that the cervix has regional patterns of loading, where the cervix is under greater load magnitudes at the internal os as compared to the external os, and the anterior/posterior regions may receive more loading depending on the patient-specific orientation of the cervical axis compared to the uterine axis (Fernandez et al. 2016, Westervelt et al. 2017).

Because clinical examination and computational studies suggest that there are functional differences within the cervix at the internal os versus the external os, the goal of the following study was to evaluate regional differences in the collagen microstructure of human NP and PG cervical tissue using secondharmonic generation (SHG) imaging. Specifically, we 
compared the collagen microstructure of samples taken from (1) different longitudinal positions: the internal os, mid-cervix and external os (Fig. 2A); (2) different radial zones: adjacent to the inner canal and an outer region comprising the middle core and outer edges of the cervix (Fig. 2D) and (3) different anatomical quadrants: anterior, posterior, left and right (Fig. 2C). Lastly, we also examined whether certain variables in a patient's obstetric history (pregnancy status, parity, number of prior vaginal deliveries (VDs)) influences collagen microstructure at the internal os, mid-cervix and external os.

SHG microscopy is a laser scanning microscopy technique similar to confocal microscopy (Zipfel et al. 2003, Williams et al. 2005), but that relies on nonlinear scattering of incident light from a specimen with an ordered structure, such as collagen. SHG imaging has been used to assess changes in collagen structure during cervical remodeling of normal pregnancy and in a PTB model in the murine cervix (Akins \& Luby-phelps 2017). This technology has also been extended to a fiber-optic scanning endomicroscopy capable of high-resolution in vivo SHG imaging of biological tissues and monitoring the remodeling of cervical collagen during gestation in mice (Zhang et al. 2012). Thus, the knowledge obtained by this study will enable (1) an improved understanding of the structure-function relationship of human cervical tissue in pregnancy and (2) discovery of non-invasive markers derived from SHG imaging that will assist in identification of cervices at risk of failure during pregnancy, leading to sPTB.

\section{Materials and methods}

\section{Sample collection and preparation}

Using a protocol approved by the Columbia University Medical Center Institutional Review Board, cervical tissue was obtained from NP and PG women (less than 50 years of age) who were undergoing hysterectomy for benign gynecologic indications or abnormal placentation respectively. All participants gave written informed consent. Three-millimeter serial transverse slices of whole cervical tissue were obtained from the level of the internal os (defined as the location at which the uterine arteries join the uterus/cervix) to the external os (Fig. 2A and B). Eight-millimeter-diameter biopsies were then taken from the inner and outer zones in each of the four quadrants (Fig. 2C and D). The anterior and posterior quadrants at the outer zone were designated as Region 1 ; the other quadrants at outer zone and all quadrants at inner zone were designated as Region 2. Each cervical tissue biopsy was immediately frozen and stored at $-80^{\circ} \mathrm{C}$ in optimal cutting temperature compound (Sakura Finetek USA, Inc., Torrance, CA, USA).
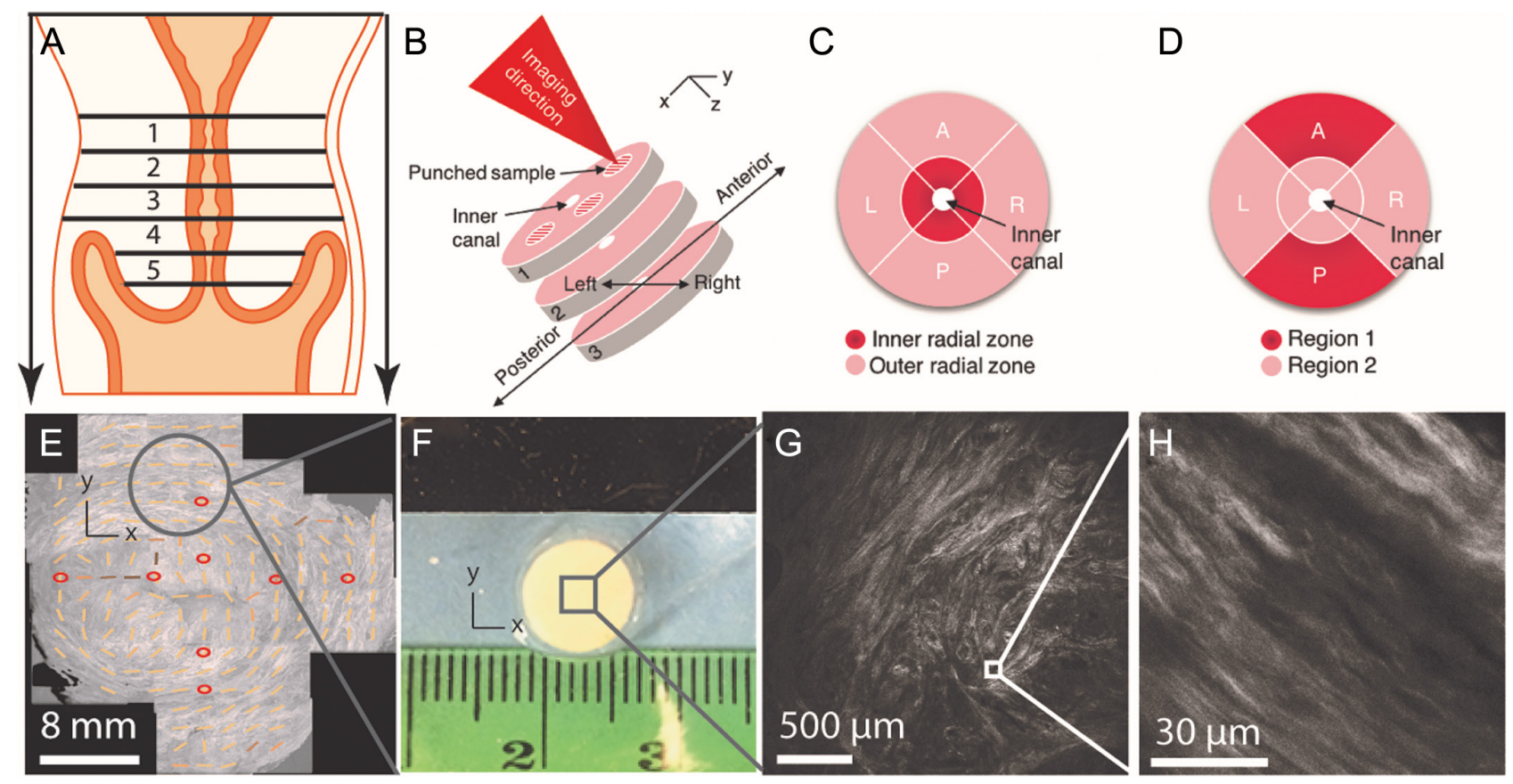

Figure 2 Methods utilized in this study. (A) Cervix slices were excised from the internal os to the external os and numbered from 1 to 5 . (B, F) $8 \mathrm{~mm}$ diameter samples were excised from each slice. SHG microscopy was used to image the samples in the $\mathrm{z}$ axis. (C) Illustration of two radial zones and quadrants. Red is the inner radial zone; pink is the outer radial zone. (D) Illustration of two anatomic regions. Red is Region 1 (anterior and posterior quadrants at the outer zone); pink is Region 2 (other quadrants at the outer zone and all quadrants from inner zone). (E) Representative OCT en face images and overlaid fiber orientation maps of a cervical slice (Yao et al. 2016). The light orange bars denote the dominant local fiber orientation in each $400 \times 400 \mu \mathrm{m}$ sub-region. Image courtesy Dr. Christine P. Hendon. Representative images of collagen microstructure are shown in (G) $2428 \mu \mathrm{m} \times 2428 \mu \mathrm{m}$, scale bar: $500 \mu \mathrm{m}$ and (H) $92 \mu \mathrm{m} \times 92 \mu \mathrm{m}$, scale bar: $30 \mu \mathrm{m}$. A, anterior; L, left; OCT, optical coherence tomography; P, posterior; R, right; $\mathrm{SHG}$, second-harmonic generation. 


\section{SHG microscopy}

Prior to SHG imaging, cervical samples were thawed, rinsed five times in $\mathrm{NaCl}$ buffer $(2 \mathrm{~mol} / \mathrm{L})$ and left to equilibrate overnight in fresh buffer at $4^{\circ} \mathrm{C}$. SHG microscopy was used to acquire images of the collagen microstructure. SHG occurs when pulsed light reacts simultaneously with the noncentrosymmetric structure of the collagen, scattering photons at twice the energy of the incident beam (Williams et al. 2005). This technique allows for optical sectioning, subsurface visualization and the use of relatively benign near-infrared laser light energies (Zipfel et al. 2003). A second-harmonic signal was generated using a Mai Tai laser (Mai Tai DeepSee, Spectra-Physics, Mountain View, CA, USA) providing a pulse width of less than $100 \mathrm{fs}$ at a $780 \mathrm{~nm}$ wavelength.

The incident beam was circularly polarized using a Berek variablewaveplate(5540, New Focus, Sanjose, CA, USA). Imaging was performed on specimens using a laser scanning confocal microscope ZEISS LSM 880 Upright (Carl Zeiss AG, Oberkochen, Germany). Consistent anatomic orientation across specimens was maintained during imaging, such that the endocervical canal was oriented parallel to the beam, with the slices excised perpendicular to the canal facing the objective (Fig. 2B). For each sample, a large representative $2427.7 \mu \mathrm{m} \times 2427.7 \mu \mathrm{m}$ region from the center of the biopsy was imaged using a $5 \times 0.25$ Fluar objective lens (Carl Zeiss) with a digital zoom of $0.7 \times$. Within the large region, three $92.4 \mu \mathrm{m} \times 92.4 \mu \mathrm{m}$ regions of interest (ROIs) were randomly selected for detailed imaging using a $20 \times 0.75$ NA Fluar objective lens (Carl Zeiss) with a digital zoom of $4.7 \times$. If an initially selected ROI fell on a large hole, a new ROI was randomly selected. At each $\mathrm{ROI}$, a z-stack was generated by acquiring images using $1-\mu \mathrm{m}$ z-increments beginning at the surface of the biopsy until no signal was detectable. Identical laser power and image acquisition parameters were used for all samples.

\section{Image analysis}

The SHG intensity signal in the SHG images was analyzed to quantify two aspects of the collagen microstructure. Because the SHG intensity scales with the square of the concentration of oriented collagen molecules (Moreaux et al. 2000), the SHG images also comprise maps of oriented collagen within the tissue. Image analysis was performed on the central $14 \mu \mathrm{m}$ (15 optical slices) of each image stack. The surface images were excluded to avoid possible tissue damage on surfaces of the biopsies that occurred during excision, and the bottom images were excluded due to signal attenuation. A custom 2D autocorrelation program (Schwille \& Haustein 2001, Rubin 2004) (IDL; Exelis Visual Information Solutions, Inc., Boulder, CO, USA) was used to perform the analysis. For each z-stack image, a 2D autocorrelation array was generated to describe the spatial similarities within the image and normalized to the maximum intensity (Fig. 3B). A 2D Gaussian surface was fit to the normalized autocorrelation array G (Fig. 3C), and two parameters were calculated to describe the collagen microstructure: the heterogeneity of intensity $\left(\mathrm{G}_{0}\right)$ and the ellipticity. The $\mathrm{G}_{0}$ term was calculated as the maximum value of the fitted Gaussian surface (Fig. 3C). A higher $\mathrm{G}_{0}$ corresponds to a greater spatial variation in SHG intensity within an image,
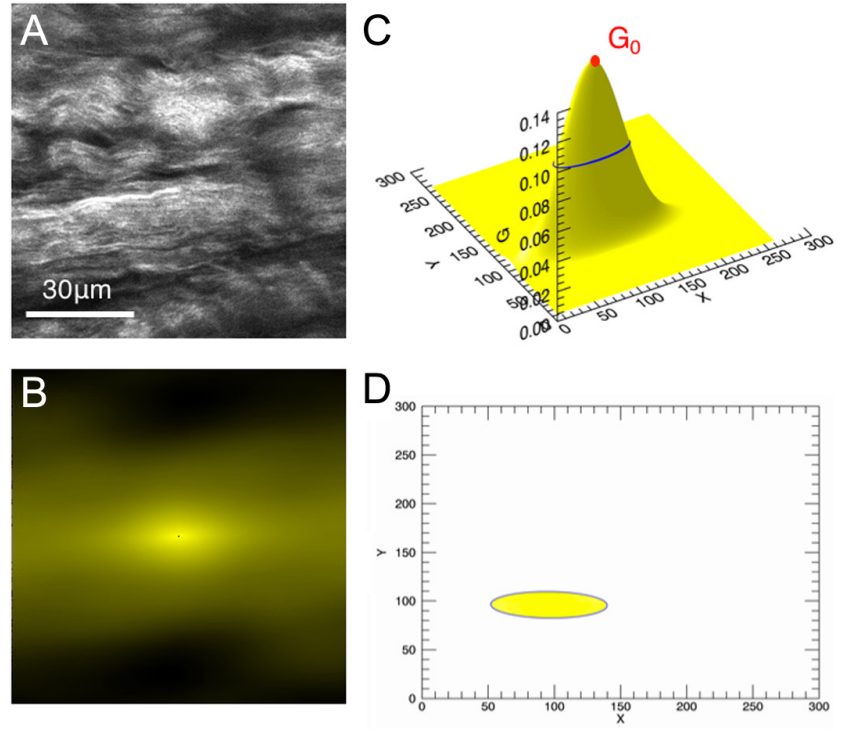

Figure 3 Illustration of 2D autocorrelation and calculation of collagen microstructure parameters. (A) The SHG image (scale bar: $30 \mu \mathrm{m}$ ) used as input for the 2D autocorrelation. (B) Map of pixel intensity from 2D autocorrelation. (C) 2D Gaussian fit of the pixel intensity map. The blue ellipse represents the fit at half of the maximum autocorrelation intensity value and the red point represents $\mathrm{G}_{0}$, the maximum value of the 2D Gaussian fit of the pixel intensity map. (D) The ellipse shown in (C) projected onto 2D $x-y$ coordinates. SHG, second-harmonic generation.

meaning that the local concentration of oriented collagen molecules is highly variable within the image (e.g. in the image at top left in Fig. 4B), whereas a lower $\mathrm{G}_{0}$ means that the local concentration of oriented collagen molecules is relatively uniform (e.g. in the image at bottom left in Fig. 4B). Ellipticity was calculated as the ratio of major to minor axes of an ellipse fit to the Gaussian surface at a height of $0.5 \mathrm{G}_{0}$ (Fig. 3C and D). Ellipticity describes the alignment of the collagen fibers, with higher values corresponding to greater collagen alignment (e.g., in the top left image in Fig. 4A), and values closer to one corresponding to disordered collagen fibers (e.g. in the bottom left image in Fig. 4B).

\section{Statistical analysis}

Ellipticity and heterogeneity of intensity $\mathrm{G}_{0}$ measured at each of the three ROls per biopsy were averaged to obtain a single value for each sample, and outcomes of samples taken from the same patient were averaged to obtain a single value for each patient. Student's $t$ tests or Wilcoxon tests (JMP, Cary, NC, USA) were used to examine the differences in microstructural parameters between groups as a function of obstetric history variables (NP versus PG, parity and number of prior VDs) and as a function of anatomic locations within the cervix (longitudinal position, radial zone and quadrant). Microstructural parameters were examined as a function of longitudinal position within the cervix from internal os (slice 1) to external os (slice 5), anatomic quadrant and parity using a one-way ANOVA. Because the current SHG analysis followed OCT (Yao et al. 2016) and histological analyses (Vink et al. 


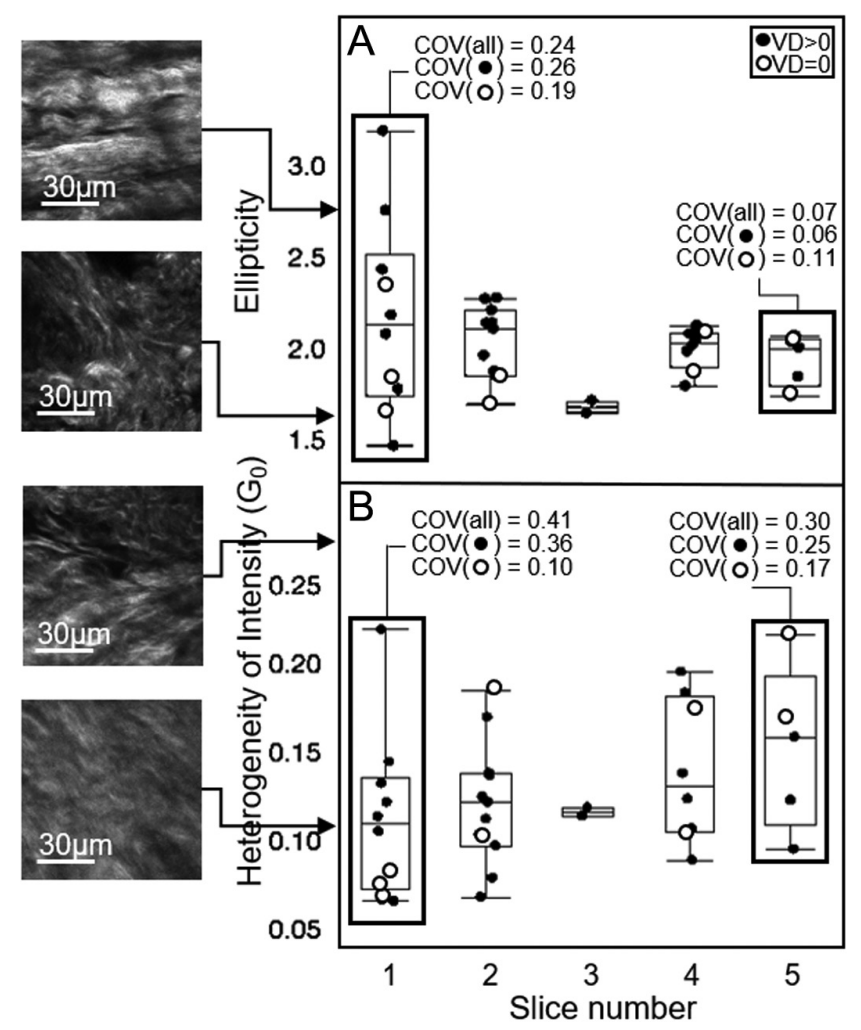

Figure 4 Cervical tissue microstructural parameters ellipticity and heterogeneity of intensity $\left(\mathrm{G}_{0}\right)$ shown as a function of longitudinal position within the cervix (slice 1: internal os; slice 5: external os) $(N=18)$. Microstructural parameters are plotted as a function of obstetric history: open symbols represent patients with zero previous vaginal deliveries $(\mathrm{VD}=0)$, while closed symbols represent patients with nonzero previous vaginal deliveries (VD>0). (A) At far left, representative SHG images of cervical tissue are shown with higher and lower values of ellipticity that fall in the range plotted in the ellipticity vs slice number plot at right. A higher ellipticity represents more-aligned fibers within the image, and a higher $\mathrm{G}_{0}$ corresponds to a greater spatial variation in SHG intensity within an image, meaning that the local concentration of oriented collagen molecules is highly variable within the image. COVs of the microstructural parameters are listed above the boxplots that are highlighted by rectangles for slices one and five. (B) At far left, representative SHG images of cervical tissue are shown with higher and lower values of heterogeneity of intensity $\left(G_{0}\right)$ that fall in the range plotted in the $G_{0}$ vs slice number plot at right. COV, coefficient of variation; SHG, second-harmonic generation.

2016) of the same cervices, variable numbers of slices from each cervix were available for analysis (Table 1). Therefore, slice number was included in the statistical analyses as a random effect to account for the differing numbers of slices analyzed for each cervix. Although the effect of position within the cervix from internal os to external os on microstructural parameters could not be examined for cervices for which only one slice was available for SHG analysis, the effects of the other variables (quadrant, radial zone, obstetric history parameters) were examined. Tukey-Kramer HSD tests were used for comparisons for pairs. The normality of the data was tested by Kolmogorov-Smirnov tests (JMP). Relationships between the microstructural outcomes and predicting variables including obstetric history variables and anatomic variables (Table 2) were examined using linear regressions. For all analyses, $P<0.05$ was considered significant.

\section{Results}

Cervical tissue was collected from 14 NP and 4 PG women. Patient obstetric histories are noted in Table 1. Patient ages ranged from 24 to 49 years, and parity ranged from 0 to 4 .

Variation in microstructural variables was examined as a function of position from the internal to external os (Fig. 2A and B). The collagen microstructure was quantified using two parameters: ellipticity, which characterizes the collagen alignment, and the heterogeneity of intensity $\left(\mathrm{G}_{0}\right)$, which characterizes the extent to which the local concentration of oriented collagen varies within an image. A higher $\mathrm{G}_{0}$ corresponds to a greater variation of oriented collagen molecules within an image. Collagen alignment assessed by ellipticity was more variable in tissue near the internal os (slice 1, coefficient of variation $(\mathrm{COV})=0.24$ ) and less variable near the external os (slice $5, \mathrm{COV}=0.07$ ) (Fig. 4A). When these parameters were further examined as a function of obstetric history, at the internal os, patients with no history of prior VDs had more uniform collagen alignment compared to those who had at least one prior vaginal delivery (VD) (COV 0.15 for $\mathrm{VD}=0$ vs 0.24 for $V D>0$ ), whereas at the external os, this difference was much less pronounced (COV 0.07 for $\mathrm{VD}=0$ vs 0.08 for $\mathrm{VD}>0$ ) (Fig. 4A). The heterogeneity of intensity exhibited a parallel trend, with values at the internal os exhibiting less variability for patients with no history of prior VDs vs those who had at least one prior VD (COV 0.10 for $V D=0$ vs 0.36 for $V D>0$ ), whereas this difference was less pronounced at the external os (COV 0.17 for $\mathrm{VD}=0$ vs 0.25 for $\mathrm{VD}>0$ ) (Fig. 4B). The COV could not be similarly assessed as a function of history of pregnancies because the patient group with no history of prior pregnancies had only one specimen available for analysis for at the external os (slice 5) (Table 1). Finally, the average values of ellipticity and heterogeneity of intensity $\left(\mathrm{G}_{0}\right)$ were similar with position from upper to lower cervix (Fig. 4A and B).

When samples were grouped based on the two zones (inner or outer radial zones) of the cervix, Fig. 2B), samples located at the inner radial zone had lower heterogeneity of intensity $\left(\mathrm{G}_{0}\right)$ than samples taken from the outer zone $(-20 \%, P=0.0460)$ (Fig. 5B). No difference in ellipticity was observed between samples from these groups (Fig. 5A). Microstructural variables were also compared between two anatomic regions within the cervix, Region 1 (anterior and posterior quadrants at outer radial zone) and Region 2 (other quadrants at outer zone and all quadrants at inner zone). These regions have been reported to vary in collagen dispersion and sensitivity to cervical remodeling during pregnancy (Yao et al. 2016). Tissue from Region 2 trended toward having lower heterogeneity of intensity 
Table 1 Patient demographics for cervical tissue.

\begin{tabular}{|c|c|c|c|c|c|}
\hline Patient number & Age (years) & Gestational age (weeks) & Previous pregnancies (G/TPAL) & \# Prior vaginal deliveries & Slices analyzed \\
\hline PG1 & 42 & 37 & $5 / 2022$ & 0 & 4,5 \\
\hline PG2 & 29 & 36 & 4/3013 & 0 & 1 \\
\hline PG3 & 35 & 36 & $3 / 2002$ & 1 & $1,2,3,4$ \\
\hline PG4 & 24 & 23 & $6 / 4014$ & 0 & 1 \\
\hline NP1 & 46 & - & 0/0000 & 0 & $2,4,5$ \\
\hline NP2 & 43 & - & 1/1001 & 1 & 2,5 \\
\hline NP3 & 49 & - & 1/1001 & 1 & 2 \\
\hline NP4 & 42 & - & 5/1041 & 1 & $1,2,4$ \\
\hline NP5 & 40 & - & $3 / 3003$ & 3 & 2,4 \\
\hline NP6 & 36 & - & 4/4004 & 0 & $2,4,5$ \\
\hline NP7 & 41 & - & $6 / 4024$ & 4 & $1,2,4,5$ \\
\hline NP8 & 41 & - & $4 / 2022$ & 0 & 2 \\
\hline NP9 & 44 & - & $3 / 3003$ & 1 & 1 \\
\hline NP10 & 38 & - & $3 / 2002$ & 0 & 1 \\
\hline NP11 & 46 & - & 2/1011 & 1 & $1,2,3,4$ \\
\hline NP12 & - & - & - & - & 1,2 \\
\hline NP13 & 36 & - & 0/0000 & 0 & 1 \\
\hline NP14 & 38 & - & $3 / 2014$ & 3 & 1 \\
\hline
\end{tabular}

G/TPAL, G= gravidity (total number of prior pregnancies); NP, non-pregnant; PG, pregnant; TPAL, term, preterm, aborted and living deliveries in the order of the four digits.

$\left(\mathrm{G}_{0}\right)$ than Region $1(-25 \%, P=0.0659)$ (Fig. 5D), but had similar ellipticity (Fig. 5C). When microstructural parameters were examined as a function of anatomic quadrants (anterior, posterior, right), no difference was observed in ellipticity or heterogeneity of intensity $\left(\mathrm{G}_{0}\right)$ (Fig. 5E and F).

Next, when collagen microstructural parameters were examined as a function of obstetric history variables, the largest difference was observed when patients were grouped by pregnancy status (NP vs PG). Specifically, ellipticity trended toward lower values in PG tissue compared to NP tissue $(-8 \%, P=0.0773)$ (Fig. 6A). Ellipticity also trended toward lower values was noted as parity increased ( $P=0.0960)$ (Fig. 6B). When comparing collagen alignment in nulliparous women vs women with one prior pregnancy, ellipticity trended toward higher values in nulliparous women $(+15 \%$ ellipticity, $P=0.0960)$. Ellipticity was similar across groups with different histories of VDs $(0$ or $>0)$ (Fig. 6C). The heterogeneity of intensity $\left(\mathrm{G}_{0}\right)$ was similar across all obstetric history groups (NP or PG; VDs 0 or $>0$;

Table 2 Variables used for linear regressions.

\begin{tabular}{|c|c|c|}
\hline Variable & Category & Values \\
\hline Ellipticity & Continuous & \\
\hline $\mathrm{G}_{0}$ & Continuous & \\
\hline Longitudinal position & Ordinal & $\begin{array}{l}\text { 0-Slice } 1 ; 1 \text {-slice } 2 ; 2 \text {-slice } 3 \text {; } \\
\text { 3-slice } 4 ; 4 \text {-slice } 5\end{array}$ \\
\hline Parity & Ordinal & Parity $=0 ;$ parity $=1 ;$ parity $>1$ \\
\hline Vaginal deliveries & Ordinal & $0-\mathrm{VD}=0 ; 1-\mathrm{VD}=1 ; 2-\mathrm{VD}>1$ \\
\hline Age (years) & Ordinal & \\
\hline Pregnancy status & Nominal & 0-NP; 1-PG \\
\hline Anatomic region & Nominal & 0-Region 1; 1-Region 2 \\
\hline Radial zone & Nominal & $\begin{array}{l}\text { 0-Inner radial zone; } 1 \text {-Outer } \\
\text { radial zone }\end{array}$ \\
\hline
\end{tabular}

Reproduction (2018) 156 71-79 parity 0,1 or $>1$ ) (Fig. 6D, E and F). When the collagen microstructural parameters within the PG group were examined as a function of gestational age, both the ellipticity values and the heterogeneity of intensity values of the PG patient with a delivery at 27 weeks (PG4) fell within the range of those with deliveries at 36-37 weeks (PG1, PG2, PG3) (Fig. 6A and D).

Finally, when relationships between microstructural SHG variables and predictors such as obstetric history variables and anatomic variables (Table 2) were examined, the singlebest predictor of ellipticity was parity. Specifically, ellipticity (collagen alignment) trended toward decreasing as parity increased $\left(R^{2}=0.28, P=0.0960\right)$. No other obstetric history variables were significant explanatory variables for the microstructural parameters examined. Anatomic variables were significantly associated with microstructural parameters. Heterogeneity of intensity $\left(\mathrm{G}_{0}\right)$ increased from inner zones to outer zones $\left(R^{2}=0.29\right.$, $P=0.0298$ ), but a similar effect on ellipticity was not observed. No other anatomic variables were significant explanatory variables for the microstructural parameters examined. Furthermore, maternal age was not a significant explanatory variable for all analyses.

\section{Discussion}

The objective of this study was to compare the collagen microstructure of cervical tissue from NP and PG women using SHG imaging to examine how human cervical collagen microstructure varies as a function of two key sets of variables (1) obstetric variables (pregnancy status, parity, number of prior VDs) and (2) anatomic location within the cervix. In general, collagen microstructural organization differed across anatomic locations within the cervix. We found that, across patients with 

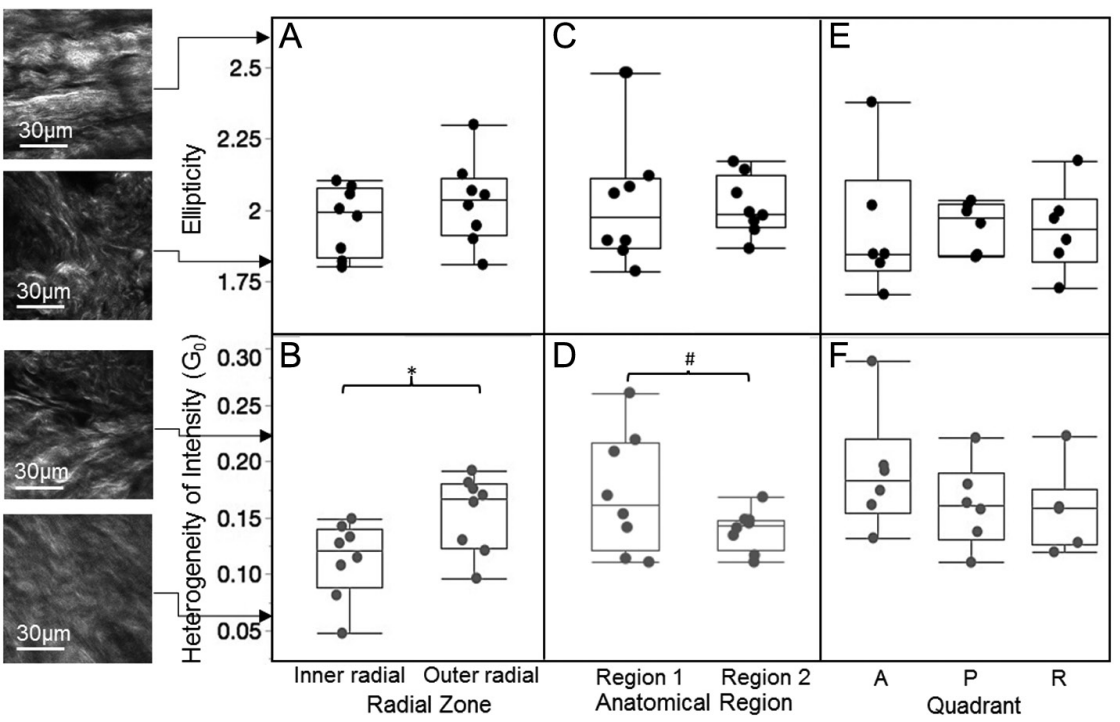

Figure 5 Collagen microstructure parameters ellipticity and heterogeneity of intensity $\left(\mathrm{G}_{0}\right)$ shown as a function of anatomic regions $(N=18)$. (A) At far left, representative SHG images of cervical tissue with higher and lower values of ellipticity that fall in the range plotted in the ellipticity vs radial zone plot. (B) At far left, representative SHG images of cervical tissue with higher and lower values of heterogeneity of intensity $\left(\mathrm{G}_{0}\right)$ that fall in the range plotted in the $\mathrm{G}_{0}$ vs radial zone plot. (C) Ellipticity vs anatomic region. (Region 1 includes anterior and posterior quadrants of the outer radial zone. Region 2 comprises all other outer radial and all inner radial zones.) (D) Heterogeneity of intensity vs anatomic region. (E) Ellipticity vs quadrant. (F) Heterogeneity of intensity $\left(\mathrm{G}_{0}\right)$ vs quadrant. ${ }^{*} P<0.05,{ }^{\sharp} P<0.10$ by Student's $t$-test or Wilcoxon rank-sum test. $\mathrm{A}$, anterior; $\mathrm{P}$, posterior; $\mathrm{R}$, right; $\mathrm{SHG}$, second-harmonic generation. different obstetric histories, collagen alignment was more variable (characterized by a higher coefficient of variance) in tissue near the internal os, which contains $50-60 \%$ cervical smooth muscle cells, and less variable (characterized by a lower coefficient of variance) near the external os, which contains only $10 \%$ cervical smooth muscle cells (Vink et al. 2016). The internal os has been suggested to be the anatomic structure most essential to normal cervical function by both MRI and ultrasound imaging (Myers et al. 2015a) owing to the fact that it is the region of the cervix that experiences the most loading in pregnancy (Fernandez et al. 2016).

Our study suggests that this difference in collagen microstructural alignment between the internal os and external os may reflect local anatomic differences in cervical function and magnitude of loading experienced in pregnancy. When collagen alignment was examined from the internal os to the external os as a function of obstetric history, patients with a history of at least one prior VD had $\sim 60 \%$ greater variation in collagen alignment at the internal os, consistent with the greater remodeling activity in response to pregnancy expected at this anatomic location. In contrast, at the external os, where less remodeling would be expected, collagen alignment had similar variation across groups with and without prior VDs. These data provide evidence of greater remodeling activity at the internal os.

Throughout the outer radial zone, cervical tissue was characterized by $20 \%$ greater spatial heterogeneity of oriented collagen molecules than that of the inner radial zone $(P<0.05)$. In particular, the anterior and posterior sections in the outer radial zone (Region 1) were characterized by $33 \%$ greater spatial heterogeneity of oriented collagen molecules than that of the rest of the

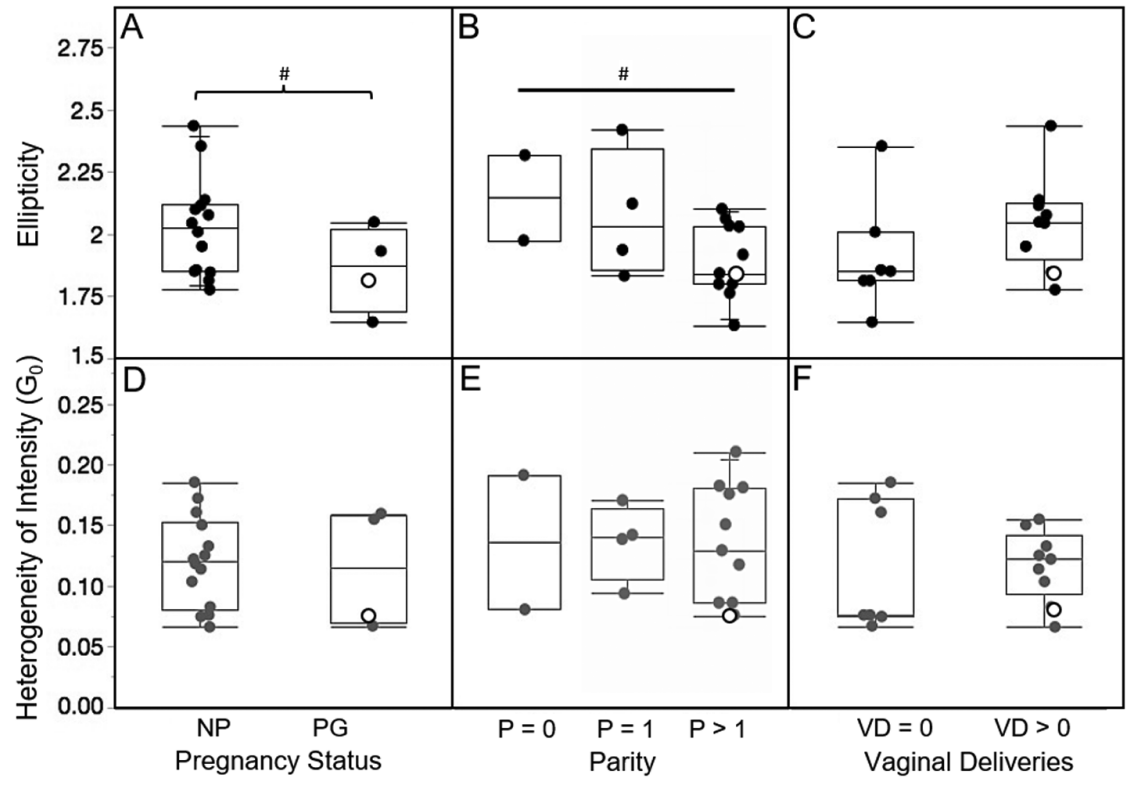

Figure 6 Cervical tissue microstructure parameters ellipticity and heterogeneity of intensity $\left(G_{0}\right)$ shown as a function of obstetric history variables $(N=18)$. (A) Ellipticity vs pregnancy status (NP and PG). (B) Ellipticity vs parity (P). (C) Ellipticity vs VDs. (D)

Heterogeneity of intensity $\left(G_{0}\right)$ vs pregnancy status. (E) Heterogeneity of intensity $\left(\mathrm{G}_{0}\right)$ vs parity. (F) Heterogeneity of intensity $\left(\mathrm{G}_{0}\right)$ Vaginal deliveries. ${ }^{*} P<0.10$ by Wilcoxon rank-sums (A) or ANOVA (B). The data point represented by an open circle corresponds to patient PG4 (delivery at 23 weeks). NP, non-pregnant; $P$, pregnant; $V D$, vaginal delivery. 
sections (Region 2), although this comparison did not reach statistical significance $(P=0.0659)$.

At the mm-scale, these regions have been reported to vary in collagen dispersion assessed by OCT where Region 1 more oriented than Region 2 (Yao et al. 2016). Interpreting both the OCT and SHG data together, at the mm-scale, Region 1 is characterized by morealigned collagen fiber bundles than Region 2; but at the micrometer length scale, the collagen fibers, on average, have a similar degree of alignment compared to those in Region 2 as assessed by the SHG ellipticity measure. These multi-scale structural data suggest that Region 1 should be stiffer in the direction of the aligned collagen fiber bundles as illuminated by the OCT (Fig. 1B). However, Region 1 is also characterized by a higher degree of heterogeneity of oriented collagen molecules compared to Region 2 as denoted by the SHG $\mathrm{G}_{0}$ measure. Although quantitative relationships between collagen microstructure and mechanical properties have not yet been established at the microscale, high values of $\mathrm{G}_{0}$ are consistent with regions of greater microscale porosity. This region-specific difference in microstructural organization may reflect an adaptive response to the local mechanical environment of the cervix. In pregnancy, the cervical axis is angled posteriorly from the uterine axis. This positioning leads to increased tissue loads and stretching in the anterior and posterior sections of the cervix (Fernandez et al. 2016) and may contribute to the difference in spatial distribution of oriented collagen molecules between Regions 1 and 2. This SHG study illuminates the need for regional mechanical tests that characterize the complex material properties of the tissue at both the mm- and micrometer-length scales to establish structure-function relationships for the human cervix.

Finally, our findings suggest that PG cervical tissue exhibits a trend toward having less-aligned collagen at the micron length scale compared to NP women. Collagen in cervical tissue from women with greater parity trended toward having decreased alignment. Previous studies using SHG imaging demonstrated that cervices from NP or gestational day 6 mice exhibit a prominent circumferential ring of aligned collagen fibers that surround a narrower zone immediately adjacent to the endocervical canal where the fibers appear more randomly arranged. In late pregnancy, this circumferential band of aligned collagen disappeared (Ambekar et al. 2012, Akins \& Luby-Phelps 2017). Our findings suggest that when compared to NP tissue, PG cervical tissue exhibits decreased collagen alignment, and this change was quantifiable by the ellipticity parameter. These findings fall in line with our OCT (Yao et al. 2016) and mechanical testing data of human NP and PG cervical tissue (Myers et al. 2015b), where a less-aligned collagen fiber of the PG cervix corresponds to a higher collagen fiber bundle dispersion on the mm-length scale and softer mechanical properties on the macroscopic length scale.

This study has key limitations and strengths. An inherent limitation of this study was that the PG samples originated from patients undergoing hysterectomy for abnormal placentation and may therefore not reflect normal remodeling, which may limit the generalizability of the results. Additionally, the sample size of PG patients is small $(N=4)$, which limits the statistical power and may limit the generalizability of the results. In addition, the PG group had a large range of gestational age: one patient delivered at 23 weeks, while the other three delivered at 36-37 weeks. Although the collagen microstructural parameters of the patient who delivered at 23 weeks were not statistical outliers and in fact fell within the range of those of the other PG patients (Fig. 6A and D), the small sample size precludes examination of the effect of gestational age on cervical remodeling. Also, our SHG imaging was only conducted on transverse 2D slices of cervix. Finally, the microstructural collagen organization along the central canal was not studied. Nevertheless, a critical strength of this study is that it provides important new information about PG and NP human cervical tissue from clinical specimens. Because most previous studies in cervical remodeling have been conducted on rodent cervices, our findings fill an important gap in knowledge about human cervical tissue in pregnancy.

Our findings elucidate outstanding questions surrounding etiologies of cervical insufficiency and PTB by providing new descriptions of the microstructural cervical collagen in NP and PG patients. This study demonstrates the ability of SHG microscopy to quantitatively evaluate the microstructure of human cervical tissue and assess premature remodeling. This study also provides direct evidence of the potential of SHG microscopy to distinguish abnormal cervical remodeling and supports future studies aimed at the development of SHG endoscopic devices for in vivo assessment of cervical properties during pregnancy. With the development of SHG imaging for clinical endoscopy (Rao et al. 2009, Zhang et al. 2012), collagen alignment, as assessed by ellipticity, shows potential as a next-generation diagnostic marker of cervical remodeling. Furthermore, in the future, the effect of pregnancy on other matrix components that affect cervical softening can be studied, including alignment and distribution of smooth muscle cells and acellular components at different anatomic locations throughout the cervix. Three-dimensional studies of collagen microstructural distribution would also be valuable due to the complexity of the structure of the human cervix. The knowledge obtained from these studies will facilitate targeted exploration of associated molecular mechanisms and lead to novel therapies for PTB.

\section{Conclusions}

When the collagen microstructure of cervical tissue from NP and PG women was examined as a function of anatomic location within the cervix and obstetric 
variables, the microstructural alignment and distribution of collagen varied with anatomic location within the human cervix. Across samples from different patients, collagen alignment was more variable near the internal os and less variable near the external os. In addition, cervical collagen alignment within the inner radial zone was more homogeneously distributed than the collagen microstructure at the outer radial zone. These observed differences in collagen microstructural alignment may reflect local anatomic differences in cervical mechanical properties and function related to pregnancy. These results contribute to a fundamental understanding of the effects of pregnancy on the evolution of the cervical microstructure and inform investigations of potential mechanisms for normal and premature cervical remodeling. Finally, our findings demonstrate the ability of SHG imaging to characterize the microstructure of human cervical tissue, provide direct evidence of the potential of SHG microscopy to distinguish abnormal cervical remodeling and support future studies aimed at the development of SHG-based diagnostic devices for in vivo assessment of cervical properties during pregnancy.

\section{Declaration of interest}

The authors declare that there is no conflict of interest that could be perceived as prejudicing the impartiality of the research reported.

\section{Funding}

This work was supported in part by NSF CMMI 1454412 to K MM.

\section{Acknowledgments}

The authors thank Beth Weed and Dr Rebecca Williams for assistance with data analysis and Dr Christine Hendon for the use of the OCT image in Fig. 2.

\section{References}

Akins ML \& Luby-phelps K 2017 Second harmonic generation imaging as a potential tool for staging pregnancy and predicting preterm birth. Journal of Biomedical Optics 15 1-10. (https://doi.org/10.1117/1.3381184)

Ambekar R, Lau T-Y, Walsh M, Bhargava R \& Toussaint KC 2012 Quantifying collagen structure in breast biopsies using second-harmonic generation imaging. Biomedical Optics Express 3 2021-2035. (https:// doi.org/10.1364/BOE.3.002021)

Fernandez M, House M, Jambawalikar S, Zork N, Vink J, Wapner R \& Myers K 2016 Investigating the mechanical function of the cervix during pregnancy using finite element models derived from high-resolution 3D MRI. Computer Methods in Biomechanics and Biomedical Engineering 19 404-417. (https://doi.org/10.1080/10255842.2015.1033163)

Mancuso MS, Szychowski JM, Owen J, Hankins G, lams JD, Sheffield JS, Perez-Delboy A, Berghella V, Wing DA \& Guzman ER 2010 Cervical funneling: effect on gestational length and ultrasound-indicated cerclage in high-risk women. American Journal of Obstetrics and Gynecology 203 259.e1-259.e5. (https://doi.org/10.1016/j.ajog.2010.07.002)
Moreaux L Sandre O \& Mertz J 2000 Membrane imaging by secondharmonic generation microscopy. Journal of the Optical Society of America B 17 1685-1694. (https://doi.org/10.1364/JOSAB.17.001685)

Myers K, Socrate S, Tzeranis D \& House M 2009 Changes in the biochemical constituents and morphologic appearance of the human cervical stroma during pregnancy. European Journal of Obstetrics and Gynecology and Reproductive Biology 144 82-89. (https://doi. org/10.1016/j.ejogrb.2009.02.008)

Myers KM, Socrate S, Paskaleva A \& House M 2010 A study of the anisotropy and tension/compression behavior of human cervical tissue. Journal of Biomechanical Engineering 132 021003. (https://doi. org/10.1115/1.3197847)

Myers KM, Feltovich H, Mazza E, Vink J, Bajka M, Wapner RJ, Hall TJ \& House M 2015a The mechanical role of the cervix in pregnancy. Journal of Biomechanics 48 1511-1523. (https://doi.org/10.1016/j. jbiomech.2015.02.065)

Myers KM, Hendon CP, Gan Y, Yao W, Yoshida K, Fernandez M, Vink J \& Wapner RJ 2015b A continuous fiber distribution material model for human cervical tissue. Journal of Biomechanics 48 1533-1540. (https:// doi.org/10.1016/j.jbiomech.2015.02.060)

Rao RAR, Mehta MR \& Toussaint KC 2009 Fourier transform-secondharmonic generation imaging of biological tissues. Optics Express 17 14534-14542. (https://doi.org/10.1364/OE.17.014534)

Robert L, Jennifer F \& Jay D 2008 Preterm birth 1: epidemiology and causes of preterm birth.

Rubin DM 2004 A simple autocorrelation algorithm for determining grain size from digital images of sediment. Journal of Sedimentary Research $\mathbf{7 4}$ 160-165. (https://doi.org/10.1306/052203740160)

Schwille P \& Haustein E 2001. Fluorescence Correlation Spectroscopy An Introduction to its Concepts and Applications. Spectroscopy 94(22).

Vink JY, Qin S, Brock CO, Zork NM, Feltovich HM, Chen X, Urie P, Myers KM, Hall TJ, Wapner R et al. $2016 \mathrm{~A}$ new paradigm for the role of smooth muscle cells in the human cervix. American Journal of Obstetrics and Gynecology 215 478.e1-478.e11. (https://doi.org/10.1016/j. ajog.2016.04.053)

Weiss S, Jaermann T, Schmid P, Staempfli P, Boesiger P, Niederer P, Caduff R \& Bajka M 2006 Three-dimensional fiber architecture of the nonpregnant human uterus determined ex vivo using magnetic resonance diffusion tensor imaging. Anatomical Record 288 84-90. (https://doi.org/10.1002/ar.a.20274)

Westervelt AR, Fernandez M, House M, Vink J, Nhan-Chang C-L, Wapner R \& Myers KM 2017 A parameterized ultrasound-based finite element analysis of the mechanical environment of pregnancy. Journal of Biomechanical Engineering 139 051004. (https://doi. org/10.1115/1.4036259)

Williams RM, Zipfel WR \& Webb WW 2005 Interpreting secondharmonic generation images of collagen I fibrils. Biophysical Journal $\mathbf{8 8}$ 1377-1386. (https://doi.org/10.1529/biophysj.104.047308)

Yao W, Gan Y, Myers KM, Vink JY \& Wapner RJ 2016 Collagen fiber orientation and dispersion in the upper cervix of non-pregnant and pregnant women. PLOS ONE 11 1-21.

Yoshida K, Mahendroo M, Vink J, Wapner R \& Myers K 2016 Material properties of mouse cervical tissue in normal gestation Mouse reproductive tract anatomy. Acta Biomaterialia 36 195-209. (https://doi. org/10.1016/j.actbio.2016.03.005)

Zhang Y, Akins ML, Murari K, Xi J, Li MJ, Luby-Phelps K, Mahendroo M \& Li X 2012 A compact fiber-optic SHG scanning endomicroscope and its application to visualize cervical remodeling during pregnancy. PNAS 109 12878-12883. (https://doi.org/10.1073/pnas.1121495109)

Zipfel WR, Williams RM \& Webb WW 2003 Nonlinear magic: multiphoton microscopy in the biosciences. Nature Biotechnology 21 1369-1377. (https://doi.org/10.1038/nbt899)

Received 15 December 2017

First decision 25 February 2018

Revised manuscript received 6 April 2018

Accepted 30 April 2018 